\title{
Tumor infiltrating immune cells (TIICs) as a biomarker for prognosis benefits in patients with osteosarcoma
}

\author{
Ying Chen ${ }^{1}$, Bo Zhao ${ }^{2}$ and Xiaohu Wang ${ }^{2^{*}}$ (D)
}

\begin{abstract}
Background: Osteosarcoma is a rare malignant bone tumor in adolescents and children. Poor prognosis has always been a difficult problem for patients with osteosarcoma. Recent studies have shown that tumor infiltrating immune cells (TIICS) are associated with the clinical outcome of osteosarcoma patients. The aim of our research was to construct a risk score model based on TIICS to predict the prognosis of patients with osteosarcoma.

Methods: CIBERSORTX algorithm was used to calculate the proportion of 22 TIIC types in osteosarcoma samples. Kaplan-Meier curves were drawn to investigate the prognostic value of 22 TIIC types. Forward stepwise approach was used to screen a minimal set of immune cell types. Multivariate Cox PHR analysis was performed to construct an immune risk score model.

Results: Osteosarcoma samples with CIBERSORTX output $p$ value less than 0.05 were selected for research. KaplanMeier curves indicated that naive B cells $(p=0.047)$ and Monocytes $(p=0.03)$ in osteosarcoma are associated with poor prognosis. An immune risk score model was constructed base on eight immune cell types, and the ROC curve showed that the immune risk score model is reliable in predicting the prognosis of patients with osteosarcoma ( $A \cup C=0.724$ ). Besides, a nomogram model base on eight immune cell types was constructed to predict the survival rate of patients with osteosarcoma.

Conclusions: TIICS are closely related to the prognosis of osteosarcoma. The immune risk score model based on TIICS is reliable in predicting the prognosis of osteosarcoma.
\end{abstract}

Keywords: Osteosarcoma, TIICs, Prognosis, Immune risk score model, Nomogram

\section{Background}

Osteosarcoma is a primary malignant bone tumor commonly seen in adolescents and children, with an incidence of 8-11 per million in the 15-19 age group [1-3]. Osteosarcoma is characterized by early lung metastasis and poor prognosis, which seriously threaten the physical and mental health of patients. Currently, chemotherapy along with surgery is the main clinical treatment,

\footnotetext{
* Correspondence: wxh18270916865@163.com

2Department of Orthopaedic, Hanchuan People's Hospital, Hanchuan 311200, Hubei Province, China

Full list of author information is available at the end of the article
}

which has improved the prognosis of osteosarcoma. However, recurrence or metastasis remain the poor prognosis [4-7]. Therefore, it is crucial to search for meaningful prognostic biomarker to improve the survival rate of patients.

Recent advances in the study of tumor microenvironment (TME) have opened up new directions for the study of osteosarcoma [8, 9]. TME is mainly composed of tumor cells, intercellular matrix, blood vessels and TIICs [10-12]. Recent studies have found a significant correlation between the TIICs in TME and prognosis in cancer patients. For example, in colorectal cancer,

(c) The Author(s). 2020 Open Access This article is licensed under a Creative Commons Attribution 4.0 International License, which permits use, sharing, adaptation, distribution and reproduction in any medium or format, as long as you give appropriate credit to the original author(s) and the source, provide a link to the Creative Commons licence, and indicate if changes were made. The images or other third party material in this article are included in the article's Creative Commons licence, unless indicated otherwise in a credit line to the material. If material is not included in the article's Creative Commons licence and your intended use is not permitted by statutory regulation or exceeds the permitted use, you will need to obtain permission directly from the copyright holder. To view a copy of this licence, visit http://creativecommons.org/licenses/by/4.0/ The Creative Commons Public Domain Dedication waiver (http://creativecommons.org/publicdomain/zero/1.0/) applies to the data made available in this article, unless otherwise stated in a credit line to the data. 
Angell $\mathrm{H}$ et al. [13] graded the density of CD3 $+\mathrm{T}$ and $\mathrm{CD} 8+\mathrm{T}$ cells at the center of the tumor and tumor infiltration border area, and added the scores to obtain the total immune score ( 0 to 4 points). Patients were divided into 5 stages according to the total immune score. Further clinical data analysis confirmed that the immune score model had a better prognostic differentiation effect than the TNM staging system. However, the construction of the model did not fully integrate with the TIICs in the tumor. Research by Zhou R et al. [14] makes up for this limitation by using the CIBERSORTX algorithm to estimate the proportion of 22 TIIC types in samples from six microarray datasets. An immune risk score model was constructed based on 22 TIIC types, which showed better prognostic value than the TNM staging system. Although a large number of studies have explored the prognostic value of TIICs in osteosarcoma [15-17], there has been no study to construct a prognostic risk score model based on immune cells. In our research, we also use CIBERSORTX algorithm to calculate the proportion of 22 TIIC types in samples from three microarray datasets containing prognostic information of osteosarcoma. An immune risk score model was constructed based on TIIC types selected by forward stepwise approach to provide more valuable biomarkers for the prognosis of osteosarcoma.

\section{Methods}

\section{Data acquisition}

To obtain gene expression microarray of osteosarcoma and corresponding clinical data, we used "osteosarcoma" and "prognosis" in the search box of the gene expression omnibus (GEO, https://www.ncbi.nlm.nih.gov/geo/) database as keywords for joint retrieval [18]. After filtering, data sets GSE16091 [19], GSE21257 [20] and GSE39055 [21] were selected as training dataset for this research. Clinical data with prognostic information were extracted directly from the matrix file on the page of the corresponding gene chip in the GEO database. Then we preprocessed the gene expression profile data from different detection platforms and converted the gene probe name into the official gene name. The testing dataset Target-OS was obtained from the Target database (https://ocg.cancer.gov/programs/target).

\section{Evaluation of TIICs}

After processing the chip, we open the CIBERSORTX website (http://CIBERSORTx.stanford.edu/), upload the processed chip data, and click to run [22-25]. At the end of the run, CIBERSORTX prints out a table containing the proportion of 22 immune cell types in each sample. CIBERSORTX will analyze each sample and calculate a $p$ value through Monte Carlo algorithm, which represents the credibility of the results. When $\mathrm{p}$ is less than 0.05 , it indicates that the proportion of 22 TIIC types analyzed by CIBERSORTX is accurate. Therefore, in this study, only samples with CIBERSORTX output $\mathrm{p}$ value less than 0.05 were selected for subsequent analysis as Christine Desmedt et al. [26] described previously. It should be noted that the output of CIBERSORTX is relative composition ratio. Therefore, the sum of the composition ratio of 22 immune cell types in each sample is 1 .

\section{Statistical analyses}

All statistical analyses in this study were carried out in $\mathrm{R}$ 3.5.3 and SPSS 23.0. All the analyses were performed by bilateral test, and a $p$ value less than 0.05 was considered statistically significant. One-way ANOVA was used for comparison between groups of continuous variables [27]. Pearson's correlation analysis and spearman's correlation analysis was used in the correlation analysis [28]. Kaplan-Meier curves were drawn to show the survival of patients, and Log-rank method was used to test whether there were differences [29]. The timedependent receiver operating characteristic (ROC) curve was used to analyze the sensitivity and specificity of the prognostic model [30]. Multivariate analysis was used to analyze the independence of immune cells as prognostic factors. Nomogram model was constructed to predict the recurrence rate of patients with osteosarcoma [31].

\section{Establishment and confirmation of an immune risk score model}

Firstly, forward stepwise approach was used to screen a more effective immune cell set at 22 TIIC types to construct a prognostic model [32]. Then, all of the selected immune cell types were fitted into multivariate Cox PHR model to establish an immune risk score model. Each sample will receive a risk score according to the immune risk score model. The samples were divided into high-risk and low-risk groups according to the cutoff value of the risk score. The Kaplan-Meier curve was drawn to compare the recurrent risk between high-risk and low-risk groups. The time-dependent receiver operating characteristic (ROC) curve to identify the reliability of the immune risk score model in predicting recurrent risk [33]. Then, we verified the model for Target-OS dataset. Patients in Target-OS dataset were divided into high-risk and low-risk groups based on the same cutoff value as GEO dataset to test the prediction ability of the immune risk score model [34].

\section{Results}

The landscape of TIICs in osteosarcoma

CIBERSORTX algorithm was used to screen out samples with CIBERSORTX output $P$ value less than 0.05 in GSE16091, GSE21257 and GSE39055 data sets for 
research. Ultimately, 95 patients with osteosarcoma was screened out (Table 1). Percentage bar chart was drawn to show the proportion of 22 TIIC types in each sample (Fig. 1a). The results revealed that the proportion of TIIC types in osteosarcoma were B cells naïve (5.14\%), B cells memory (0.88\%), Plasma cells (1.77\%), T cellsCD8(6.43\%), T cells CD4 naive (0.90\%), T cells CD4 memory resting (0.74\%), T cells CD4 memory activated (1.02\%), T cells follicular helper (4.79\%), Tregs (2.40\%), $\mathrm{T}$ cells gamma delta (6.02\%), NK cells resting (0.38\%), NK cells activated (2.26\%), Monocytes (1.53\%), Macrophages M0 (40.54\%), Macrophages M1 (3.66\%), Macrophages M2(13.34\%), Dendritic cells resting (1.40\%), Dendritic cells activated (0.79\%), Mast cells resting (2.88\%), Mast cells activated (2.43\%), Eosinophils $(0.05 \%)$, Neutrophils $(0.62 \%)$, respectively. Figure $1 \mathrm{~b}$ indicated the correlation coefficient between 22 TIIC types. Red and blue colors indicate positive and negative correlation, respectively. Color intensity corresponds to the degree of correlation. We can find that eosinophils and memory B cells have the strongest positive correlation $(r=0.61), \mathrm{CD} 8+\mathrm{T}$ cells had the strongest negative correlation with M0 Macrophages and $\gamma \delta \mathrm{T}$ cells $(r=-0.54)$.

\section{Predictive value of TIICs in osteosarcoma}

To investigate the prognostic value of 22 TIIC types, Kaplan-Meier curves were drawn and the results were evaluated by log-rank test. In Fig. 2, we can find that high abundance of naive $\mathrm{B}$ cells $(p=0.047$, Fig. 2a) and Monocytes ( $p=0.03$, Fig. $2 \mathrm{~b})$ in osteosarcoma is associated with poor prognosis.

\section{Establishment and confirmation of an immune risk score model}

Considering that the effect of TIICs on the prognosis of patients is not independent. We constructed an immune risk score model to evaluate the prognosis of patients individually. Firstly, we used the forward stepwise regression method to screen out a minimal set of TIIC types. Finally, eight immune cell types were selected. Then, eight immune cell types were filled into multivariate Cox PHR analysis to construct an immune risk score model. Formula is as follows: Risk $_{8}=8.49$ * naive B cells +15.7 * activated memory CD $4+\mathrm{T}$ cells $+17.1 *$ follicular helper $\mathrm{T}$ cells $+6.07 * \gamma \delta \mathrm{T}$ cells $+27.9 *$ resting Dendritic cells $+24.7 *$ activated Dendritic cells - $26.8 *$ Neutrophils -

Table 1 The proportional distribution of samples with different $P$-value in three datasets

\begin{tabular}{llll}
\hline & $\boldsymbol{P}<\mathbf{0 . 0 5}$ & $\boldsymbol{P}>\mathbf{0 . 0 5}$ & Total \\
\hline GSE16091 & 33 & 1 & 34 \\
GSE21257 & 52 & 1 & 53 \\
GSE39055 & 10 & 27 & 37 \\
Total & 95 & 29 & 124 \\
\hline
\end{tabular}

7.38 * activated Mast cells (Table 2). Each sample will be given a risk score based on the model. Patients were divided into high- and low-risk group according to the median risk score. Kaplan-Meier curves indicated that patients in high-risk group had a poorer prognosis than those in low-risk group $(p=8.303 \mathrm{e}-04$, Fig. 3a). The ROC curve showed that the immune risk score model is reliable in predicting the prognosis of patients with osteosarcoma (AUC $=0.724$, Fig. 3b). Principal component analysis revealed that the immune risk score model can distinguish the patients into high - and low-risk groups well (Fig. 3c). In addition, Fig. 3d, e and $f$ respectively showed the risk score, survival status and 8 immune cell types infiltration of patients with osteosarcoma. Then, the prediction ability of the immune risk score model was verified in Target-OS dataset based on the same cutoff value as GEO dataset. The result indicated that the prediction ability of the immune risk score model is reliable (Fig. 4).

\section{Independent predictive power of eight immune cell types} We also used multivariate analysis to explore whether these 8 immune cell types were independent prognostic factors for osteosarcoma patients. The results indicated that naive B cells, activated memory CD4 + T cells, follicular helper $\mathrm{T}$ cells, resting dendritic cells, activated dendritic cells were independent predictors for osteosarcoma patients (Fig. 5).

\section{Construction of nomogram model}

To assess 1, 3, 5-year survival in patients with osteosarcoma, we constructed a nomogram based on eight screened immune cell types. In the nomogram model, there are several vertical lines passing through the value of each variable, and different integrals can be obtained on the integral line at the top of the nomogram. The integrals of all the variables are added to obtain the total score, and the predicted survival probability values at the corresponding time points are calculated from the line perpendicular to the total score on the prediction line at the bottom of the nomogram. Probability of death $=1$ probability of survival. According to the nomogram, we can score the influence of eight immune cell types on the prognosis of patients, and then use the total score to evaluate the 1,3,5-year survival rate of patients with osteosarcoma (Fig. 6a). The calibration curve revealed that the predictive ability of the nomogram model at 1-, 3- and 5-year is accurate (Fig. 6b, c, d). The DCA curve revealed that the nomogram model can benefit for the patients (Fig. 6e). Clinical impact curve found that the predictive power of the nomogram model is remarkable (Fig. 6f). 


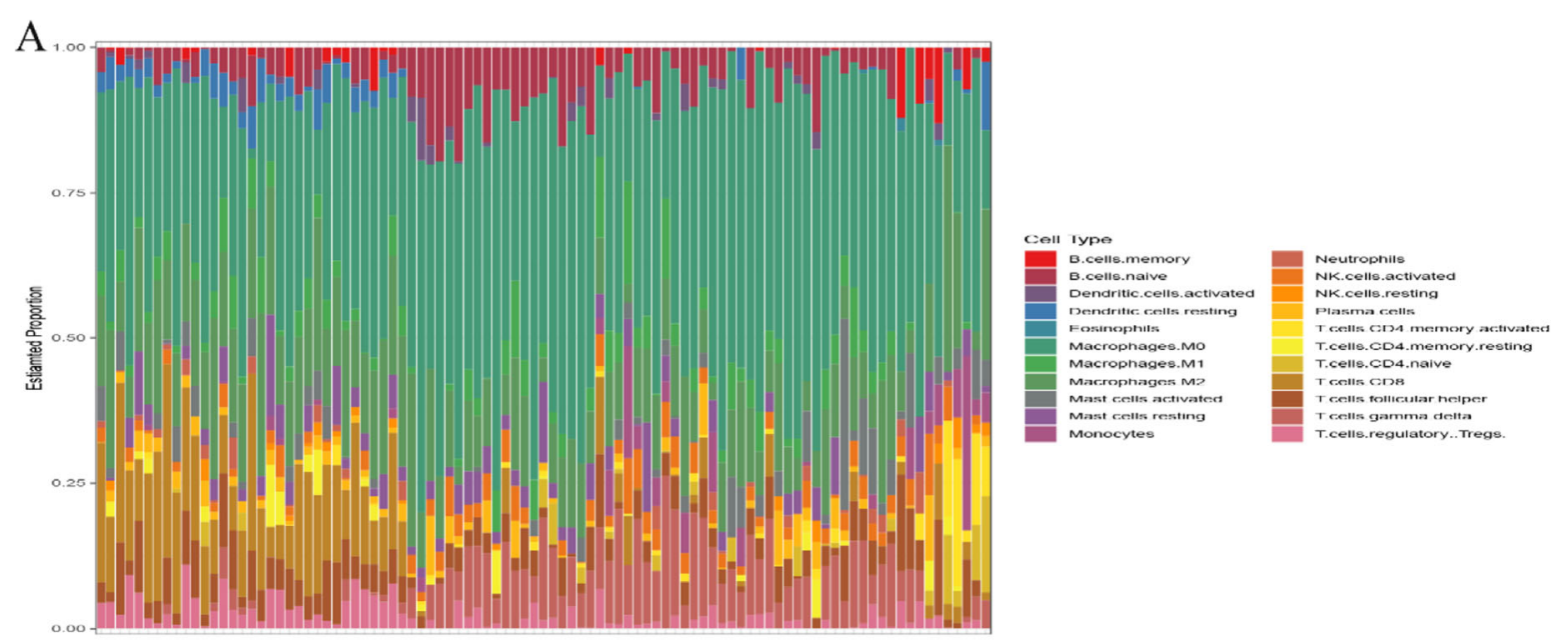

B

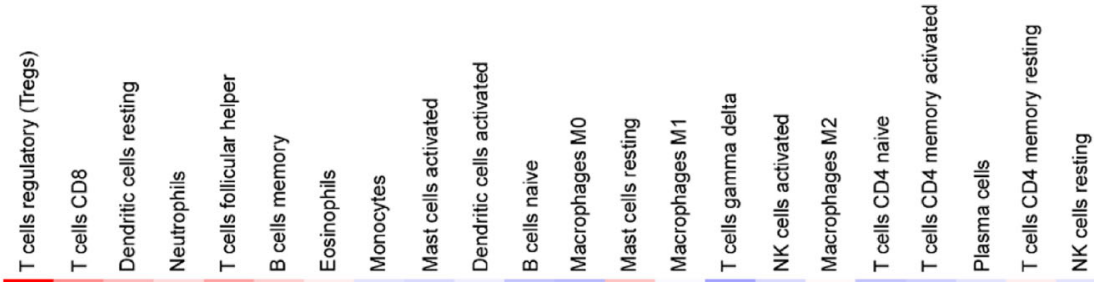

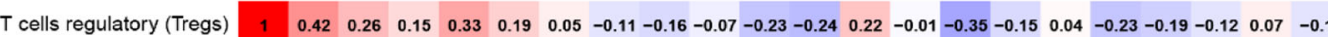

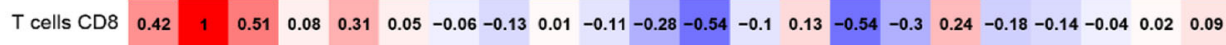

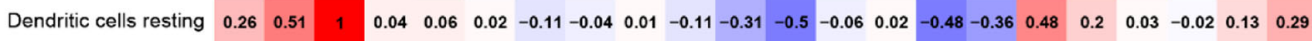

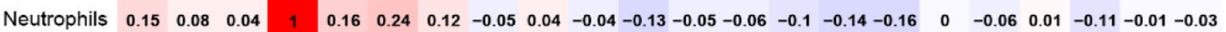

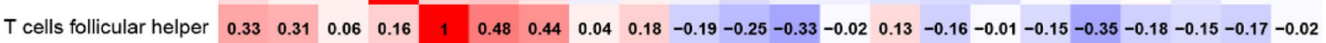

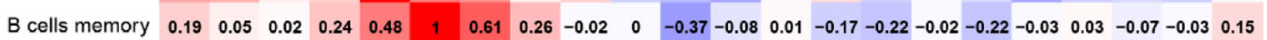

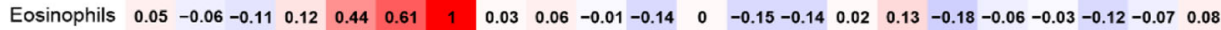

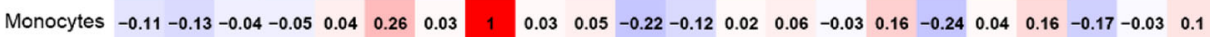
$\begin{array}{llllllllllllllllllllllllllllllllllll}\text { Mast cells activated } & -0.16 & 0.01 & 0.01 & 0.04 & 0.18 & -0.02 & 0.06 & 0.03 & 1 & -0.12 & -0.19 & -0.08 & -0.4 & 0.05 & -0.03 & -0.03 & -0.16 & 0.04 & 0.04 & -0.09 & -0.06 & 0.02\end{array}$

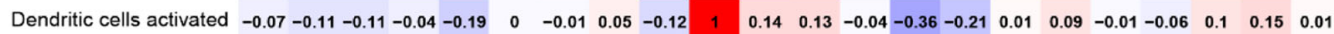

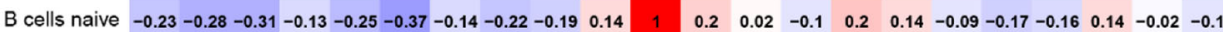

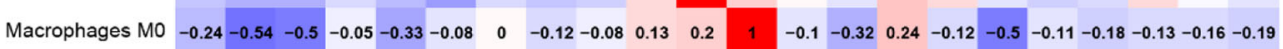

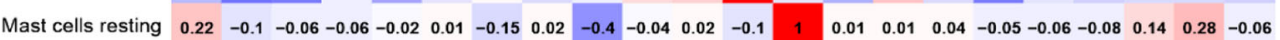

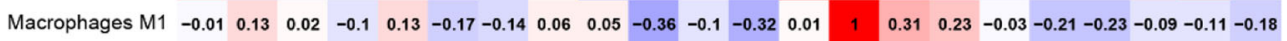

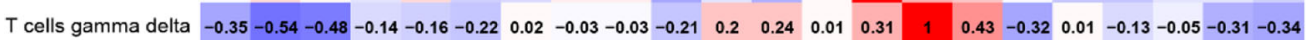
$\begin{array}{llllllllllllllllllllllll}\text { NK cells activated } & -0.15 & -0.3 & -0.36 & -0.16 & -0.01 & -0.02 & 0.13 & 0.16 & -0.03 & 0.01 & 0.14 & -0.12 & 0.04 & 0.23 & 0.43 & 1 & -0.13 & -0.08 & -0.06 & 0.1 & 0.02 & -0.31\end{array}$

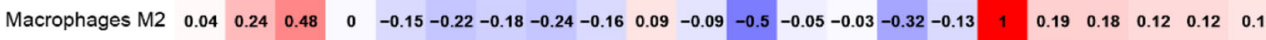

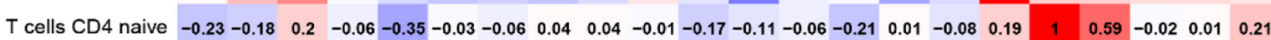
$\begin{array}{llllllllllllllllllllllll}\text { T cells CD4 memory activated } & -0.19 & -0.14 & 0.03 & 0.01 & -0.18 & 0.03 & -0.03 & 0.16 & 0.04 & -0.06 & -0.16 & -0.18 & -0.08 & -0.23 & -0.13 & -0.06 & 0.18 & 0.59 & 1 & -0.08 & 0.04 & 0.44\end{array}$

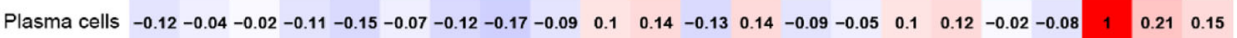
$\begin{array}{llllllllllllllllllllllllllllll}T \text { c cells CD4 memory resting } & 0.07 & 0.02 & 0.13 & -0.01 & -0.17 & -0.03 & -0.07 & -0.03 & -0.06 & 0.15 & -0.02 & -0.16 & 0.28 & -0.11 & -0.31 & 0.02 & 0.12 & 0.01 & 0.04 & 0.21 & 1 & 0.32\end{array}$

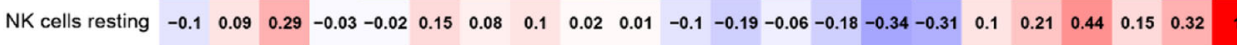

Fig. 1 The landscape of tumor-infiltrating immune cells in osteosarcoma. a. The proportion of 22 immune cell types in osteosarcoma tissues. $\mathbf{b}$. Correlation matrix between 22 immune cell types in osteosarcoma 

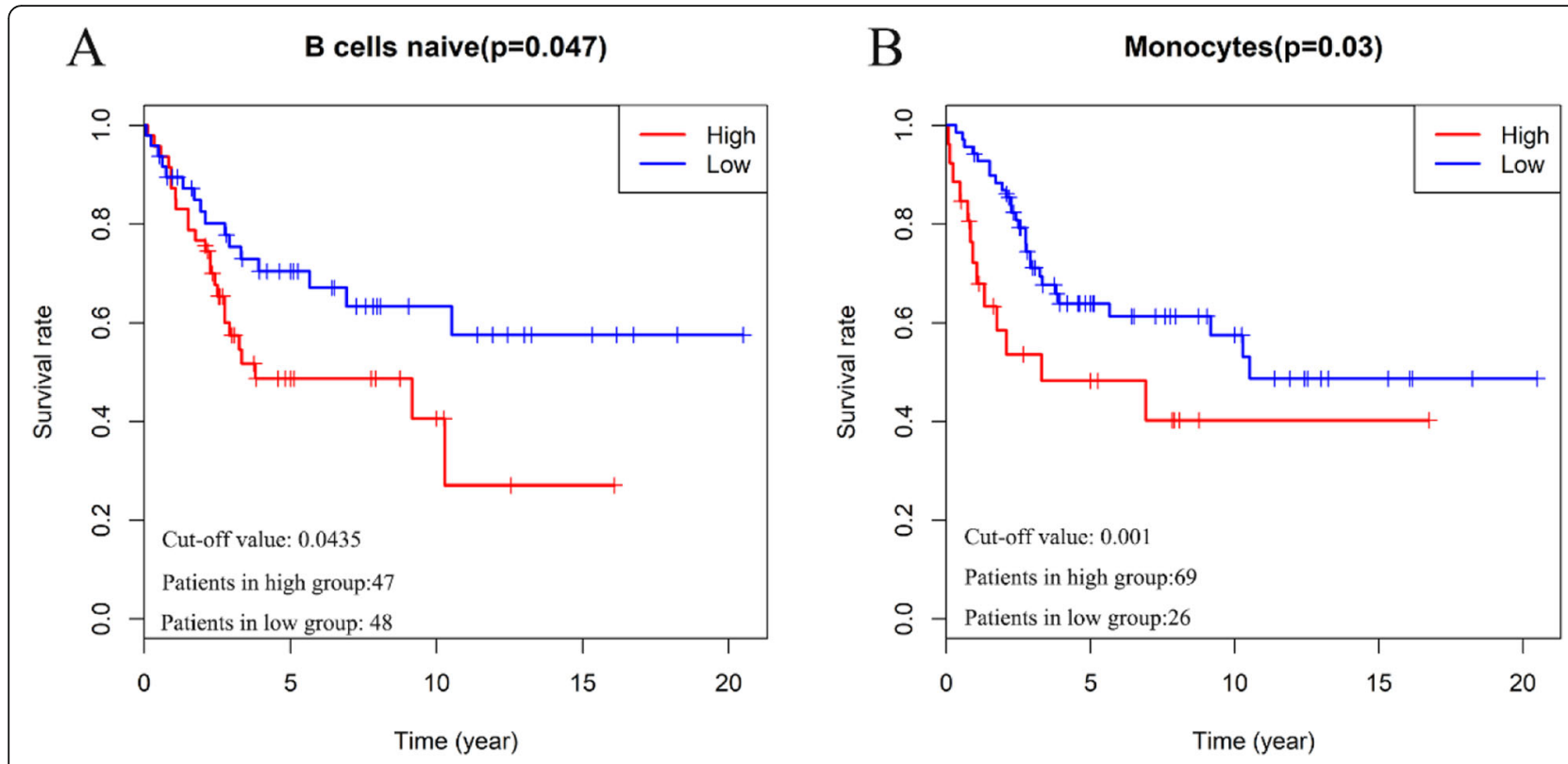

Fig. 2 The prognostic value of 22 immune cell types in osteosarcoma by plotting Kaplan-Meier curves

\section{Biological phenotypes related to immune risk score}

We explored the expression data in testing dataset and evaluated the correlation between immune risk score and biological phenotypes (immune checkpoint, cell proliferation and DNA repair). We found that the patients in the high risk group had significantly lower expression levels of immune checkpoint related genes than in the low risk group, which indicated that the patients in the low risk group may sensitive to immunotherapy. However, the genes related to cell proliferation and DNA repair were higher expressed in the high risk group (Fig. 7).

\section{Discussion}

Recurrence and metastasis are the main reasons for poor prognosis of osteosarcoma. It is of great significance to accurately predict the recurrence risk of patients and carry out appropriate monitoring and intervention for patients with high recurrence risk. In recent years, increasing researchers began to pay attention to the role of immune microenvironment in tumor development. A recent meta-analysis included more than 120 literature, systematically summarizing the effects of various immune cells such as B cells, NK cells, macrophages and all $\mathrm{T}$ cell subsets on the clinical outcomes of tumor patients. The results showed that cytotoxic $\mathrm{T}$ cells and memory $\mathrm{T}$ cells were beneficial for survival in different tumor types, while the prognosis effect of other immune cells such as B cell, NK cell, macrophages and some helper $\mathrm{T}$ cell subsets are associated with tumor type and stage [35]. However, the comprehensive analysis of 22 TIIC types in osteosarcoma is still unsatisfactory. Zhang $C$ et al. [5] used ESTIMATE algorithm to obtain an immune score for each osteosarcoma case from TCGA database, and osteosarcoma cases were divided into the high or low score groups. Difference analysis showed that M0 macrophages and naive B cells were lower in

Table 2 Multivariate Cox PHR analysis of eight immune cells. We found that naive B cells, activated memory CD $4+T$ cells, follicular helper T cells, resting Dendritic cells, activated Dendritic cells were independent predictors for osteosarcoma patients $(P<0.05)$

\begin{tabular}{|c|c|c|c|c|c|}
\hline & coef & $\exp ($ coef) & se (coef) & $\mathbf{z}$ & $\mathbf{p}$ \\
\hline B.cells.naive & $8.49 \mathrm{E}+00$ & $4.87 E+03$ & $3.47 E+00$ & 2.451 & 0.014255 \\
\hline T.cells.CD4.memory.activated & $1.57 E+01$ & $6.55 E+06$ & $5.18 \mathrm{E}+00$ & 3.031 & 0.002435 \\
\hline T.cells.follicular.helper & $1.71 E+01$ & $2.61 E+07$ & $5.12 \mathrm{E}+00$ & 3.334 & 0.000855 \\
\hline T.cells.gamma.delta & $6.07 E+00$ & $4.32 E+02$ & $3.37 \mathrm{E}+00$ & 1.799 & 0.071947 \\
\hline Dendritic.cells.resting & $2.79 E+01$ & $1.29 E+12$ & $8.71 E+00$ & 3.203 & 0.001359 \\
\hline Dendritic.cells.activated & $2.47 E+01$ & $5.33 \mathrm{E}+10$ & $9.45 \mathrm{E}+00$ & 2.614 & 0.008949 \\
\hline Neutrophils & $-2.68 \mathrm{E}+01$ & 2.27E-12 & $1.82 \mathrm{E}+01$ & -1.472 & 0.1411 \\
\hline Mast.cells.activated & $-7.38 \mathrm{E}+00$ & $6.21 \mathrm{E}-04$ & $6.09 E+00$ & -1.212 & 0.225384 \\
\hline
\end{tabular}




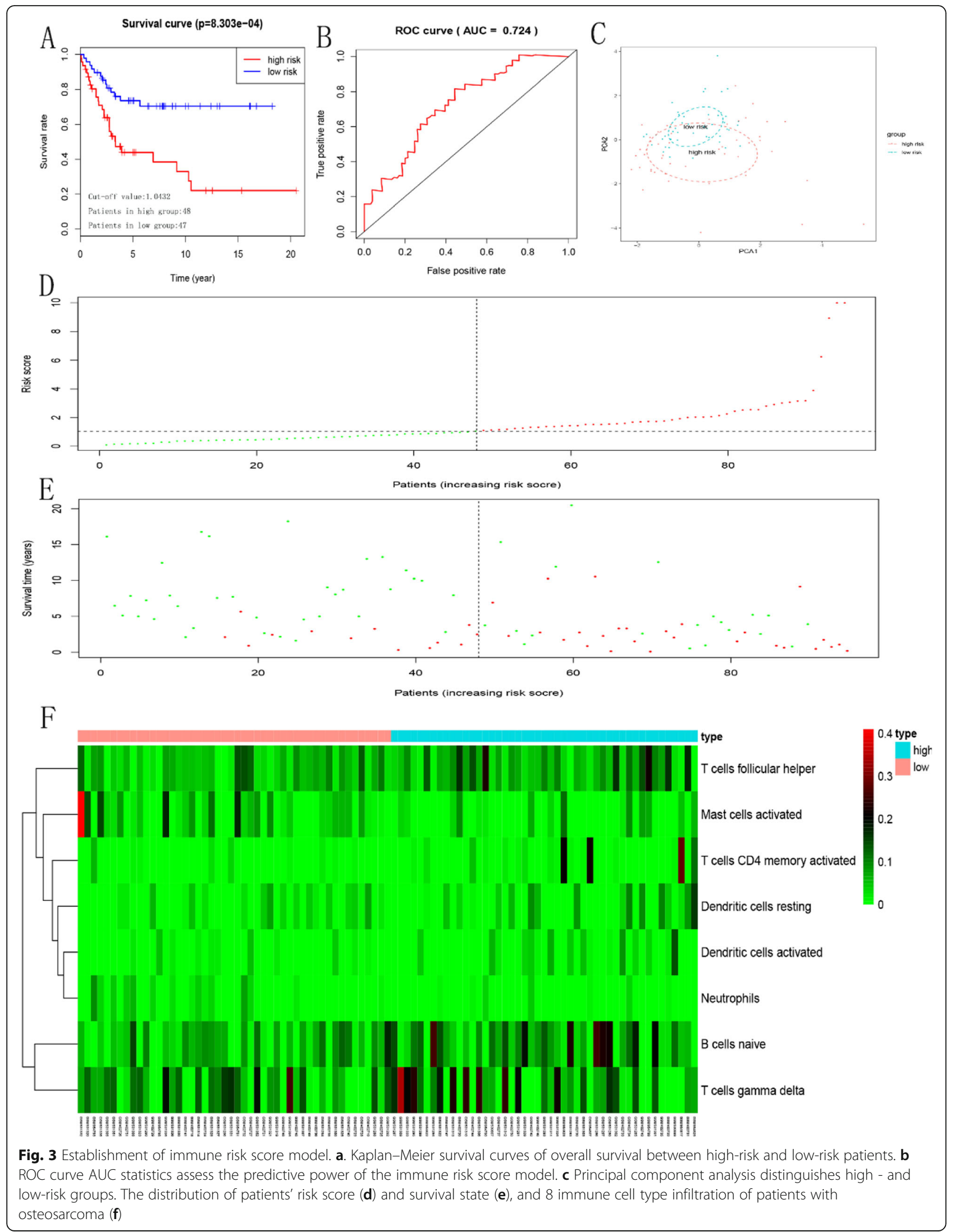




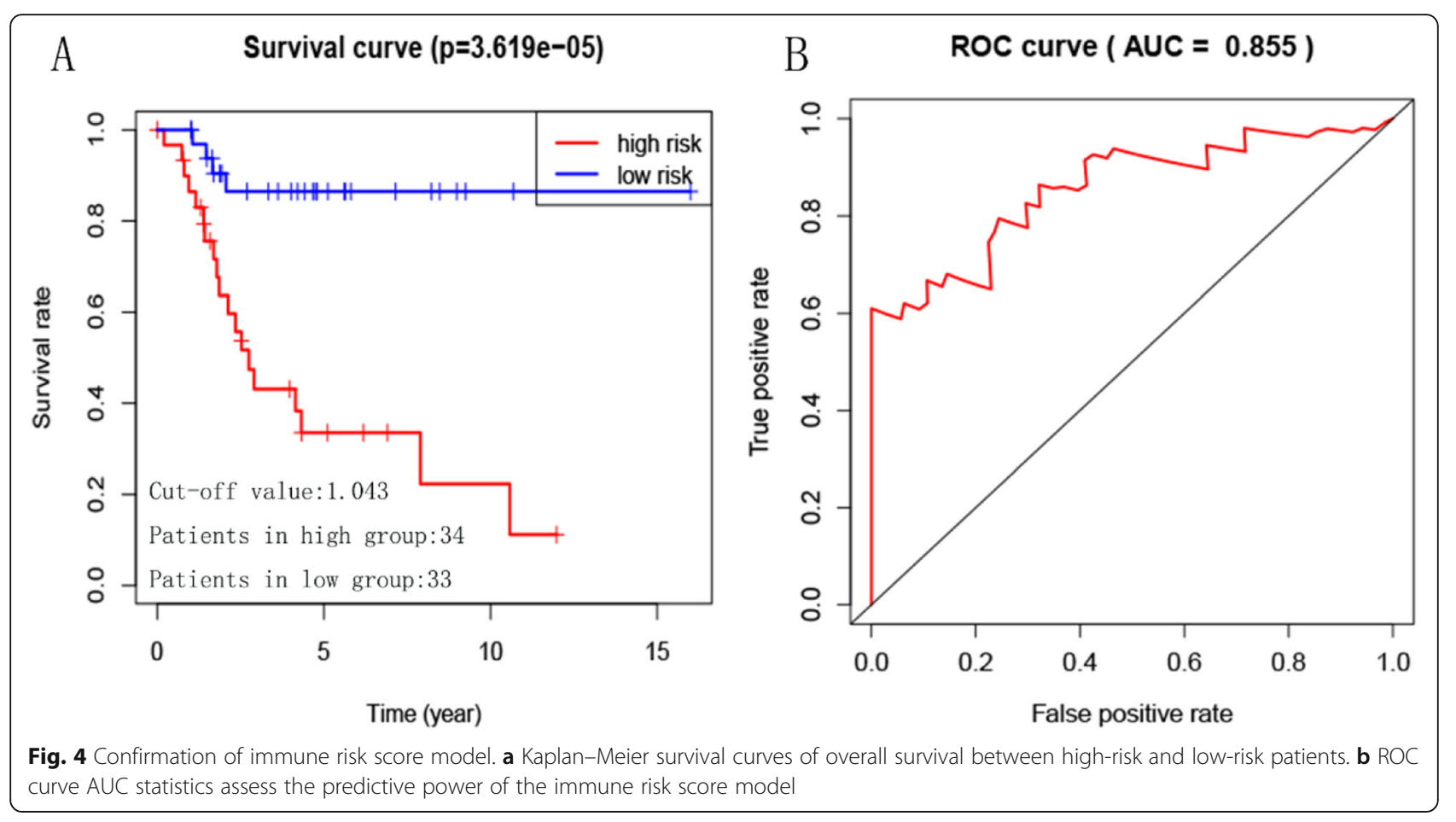

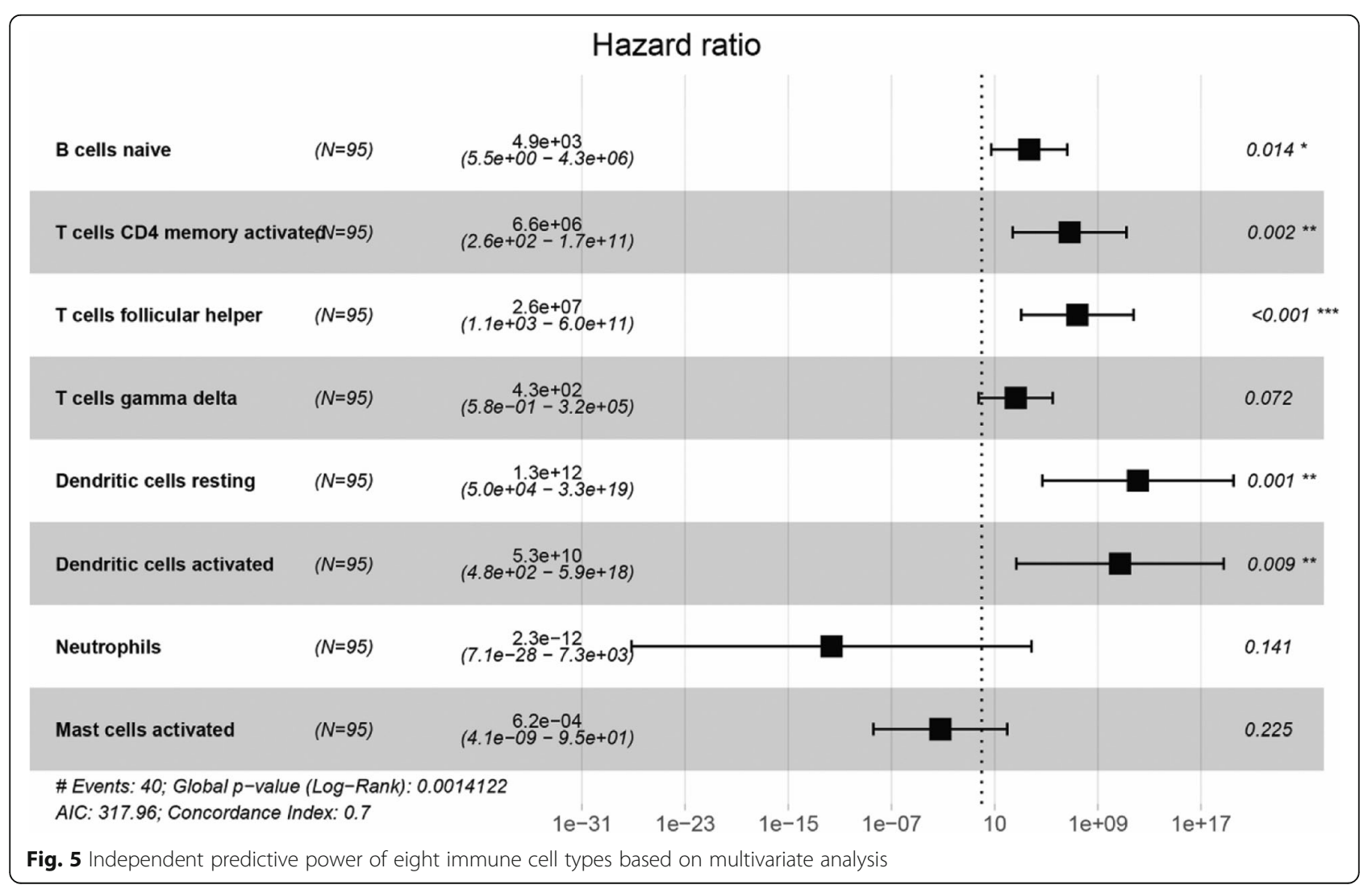




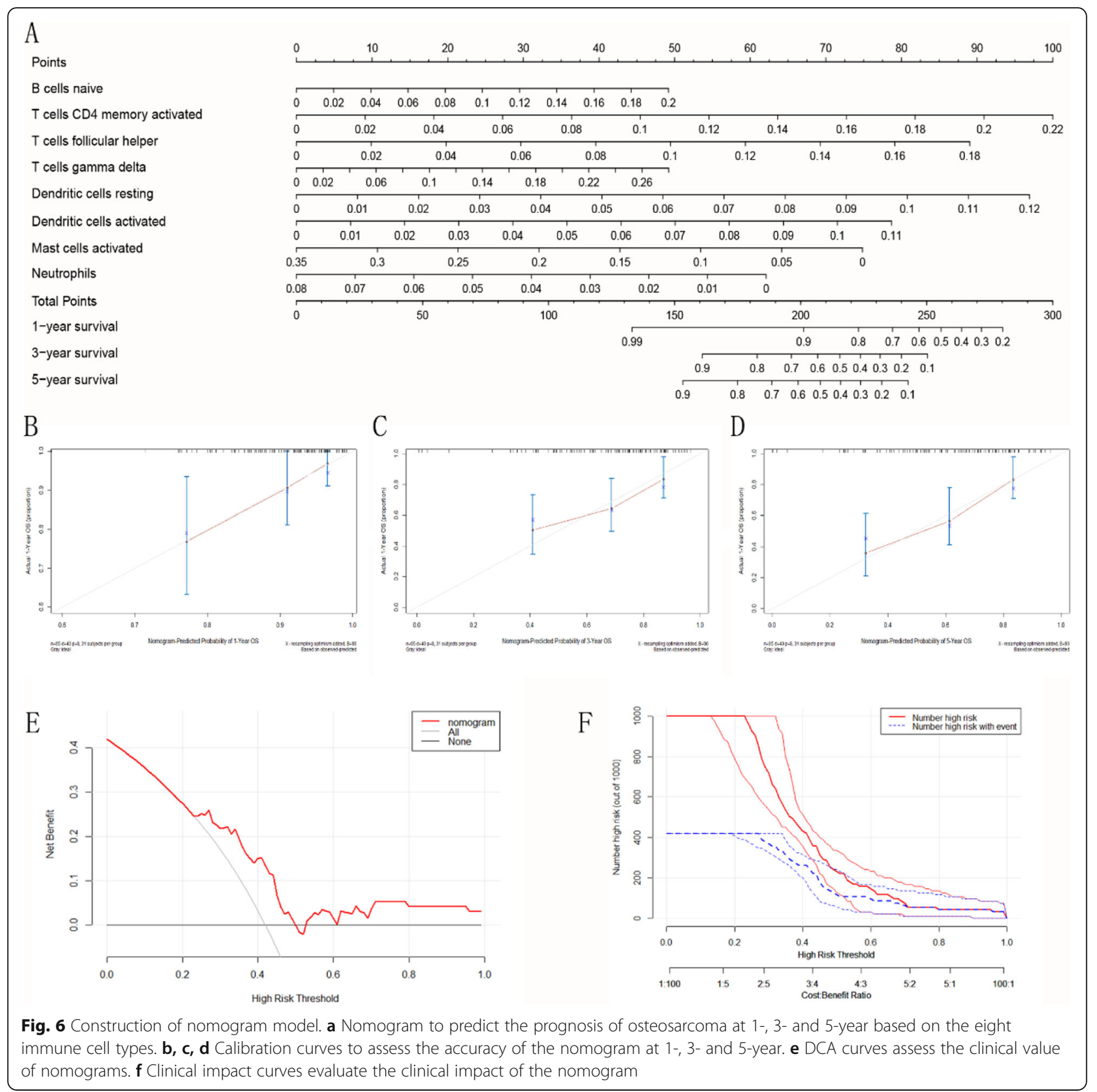

high immune score group than in low immune score group, while M1 macrophages, resting dendritic cells, and M2 macrophages were higher. Then, a risks core model based on different immune related genes was established to predict the prognosis of osteosarcoma. Weifeng Hong et al. [36] used the similar method as Zhang $\mathrm{C}$ to investigate the immune infiltration of osteosarcoma from Target database. The result indicated that $\mathrm{T}$ cells CD8, $\mathrm{T}$ cells CD4 memory activated, M1 macrophages and M2 macrophages were higher in high immune score group than in low immune score group, while Plasma cells, T cells CD4 naive $(0.90 \%), \mathrm{T}$ cells
CD4 memory resting, M0 macrophages and Mast cells resting were lower. Deng $C$ et al. [37] used CIBERSORTX algorithm explored the changes of 22 immune cell types infiltration of osteosarcoma from Target database after neoadjuvant chemotherapy. The result indicated that $\mathrm{CD} 8+\mathrm{T}$ cells, CD3+ T cells, $\mathrm{PD}-\mathrm{L} 1+$ immune cells and Ki67 + CD8 + T cells in osteosarcoma increased after neoadjuvant chemotherapy. In our research, CIBERSORTX algorithm was used to analyze gene expression in osteosarcoma and obtain the proportion of 22 TIIC types. The results revealed that macrophages account for a large proportion of 22 immune cell types, 


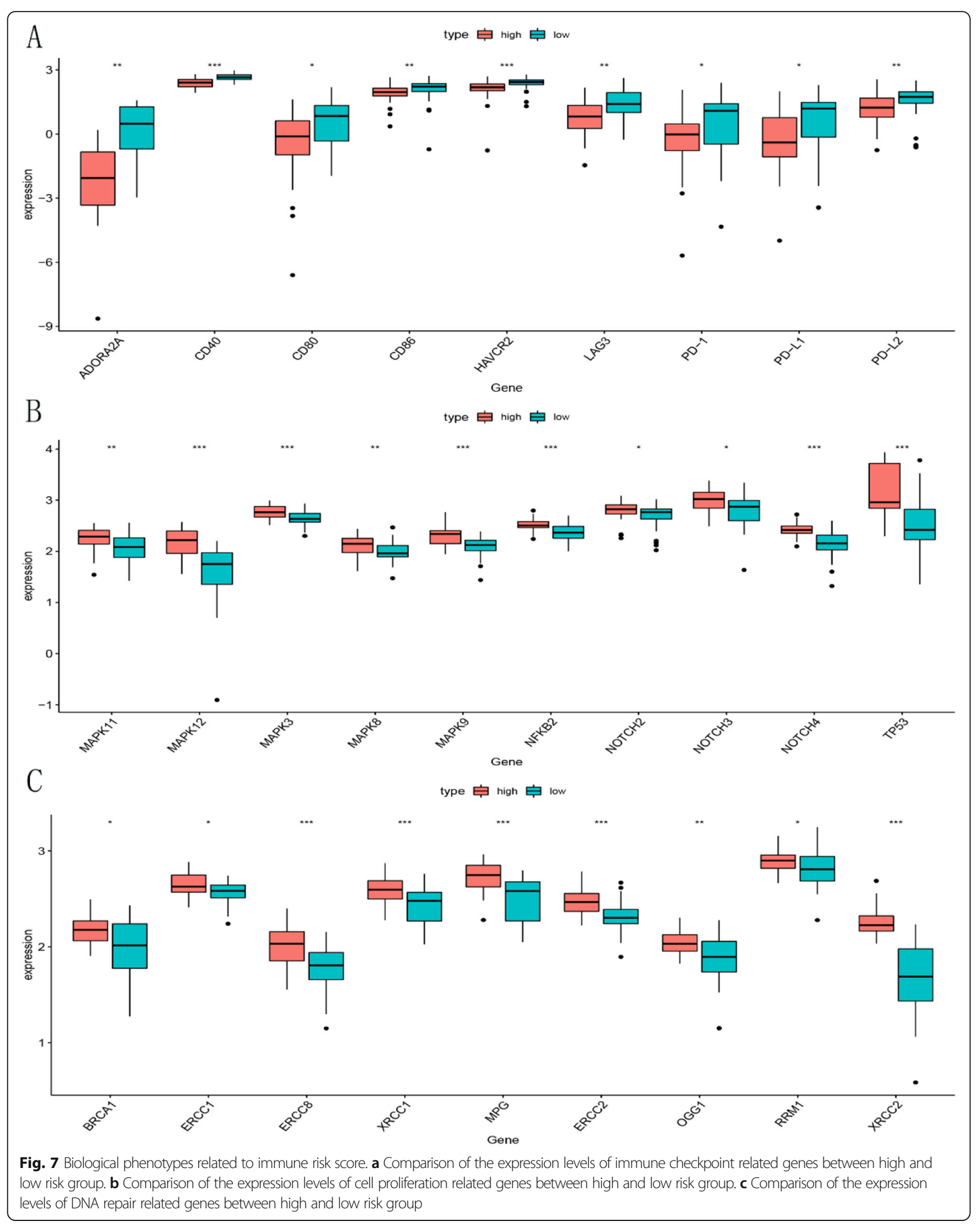


which were consistent with Morrison C's study [38]. Then Kaplan-Meier curves were drawn to investigate the prognostic value of 22 TIIC types. The results indicate that naive $\mathrm{B}$ cells and Monocytes are associated with poor prognosis of osteosarcoma. As an important component of humoral immunity, B lymphocytes can exert a two-way role in tumor. B lymphocytes can positively regulate tumor immune process by producing antitumor antibodies, secreting various cytokines, and acting as antigen-presenting cells, and negatively regulate tumor immune process by inhibiting the proliferation of immune-activated $\mathrm{T}$ cells [39]. In addition, studies have shown that elimination of B lymphocytes not only helps to inhibit tumor progression and recurrence, but also significantly increases the sensitivity of patients to chemotherapy [40]. As the precursor of macrophages, monocytes can be recruited into tumor tissues and polarized into M1 macrophages or M2 macrophages in different tumor environments [41]. Sottnik JL [42] and Tuohy JL [43] all revealed that monocytes are negatively associated with disease free survival of canine osteosarcoma. Cersosimo F. et al. reviewed that M2 macrophages was associated with OS metastasis and poor patient prognosis [44], which partly support our analysis results.

At present, several immune risk score models used to quantify the immune status and indicate the prognosis of patients have been proposed in colorectal cancer and breast cancer respectively. These immune risk score models was proposed based on the counts of two lymphocyte populations of tumor centers and invasion margins $[45,46]$. The immune risk score model can be used to identify patients with recurrent risk or benefit from corresponding adjuvant chemotherapy. However, there have no studies involve the construction of immune risk score model in osteosarcoma. In our study, we constructed an immune risk score model based on eight immune cell types selected by forward stepwise approach from 22 TIIC types. Different from the traditional immunohistochemical method, the immune cells we used to construct the immune risk score model was screened by CIBERSORTX algorithm. It has more advantages than traditional methods, such as simple operation, accurate results and more immune cell markers. As expecting, our immune risk score model showed a well prognostic value. The patients in the high risk group had a poor prognosis may relate to stronger cell proliferation and repair ability. In conclusion, the immune risk score model has a good clinical application value and worth popularizing. In addition, considering the intuition of the immune risk score model in clinical application, we constructed a nomogram model based on the eight selected immune cell types to present the prediction results simply and clearly. Nomogram is a model that quantifies the probability of an event individually and accurately by integrating multiple predictors based on multi-factor analysis. So far, there is no perfect nomogram model, and the nomogram model in this study can only serve as a reference for clinicians.

Some limitations of this research should be discussed. Firstly, without complete clinical information, in addition to survival and follow-up data, other important clinical information such as age, gender, staging, metastasis and chemotherapy are not available, so that we cannot explore the independence of the prognostic value of the immune risk score model. Besides, it is also impossible to compare the immune risk score model with traditional GTM stage. Secondly, the heterogeneity of the location of immune infiltration was not taken into account in this research. Thirdly, samples were downloaded from different datasets to enlarge the sample size, which may influent the repeatability of outcome. Finally, limited prognostic osteosarcoma data can be searched in the GEO database, so there may be some bias in the results.

\section{Conclusions}

In summary, our study revealed an association between clinical outcomes and immune infiltration patterns in osteosarcoma. The immune risk score model based on eight immune cell types is reliable in predicting the prognosis of osteosarcoma.

\section{Abbreviations}

TME: Tumor microenvironment; Cox PHR: Cox proportional hazard regression model; ROC: Receiver operating characteristic curve; TIICs: Tumor infiltrating immune cells; GEO: Gene expression omnibus

\section{Acknowledgments}

We thank the authors who provided the GSE16091, GSE21257 and GSE39055 database.

\section{Authors' contributions}

YC, BZ, XW conceived and designed the study. YC, BZ, XW developed the methodology. YC, XW analyzed and interpreted the data. YC wrote, reviewed, and/or revised the manuscript. All authors have read and approved the manuscript.

\section{Funding}

Not applicable.

\section{Availability of data and materials}

All data generated or analysed during this study are included in this published article and its supplementary information files. GSE16091, GSE21257 and GSE39055 dataset was downloaded from GEO database (https://www.ncbi.nlm.nih.gov/geo/). The testing dataset Target-OS was obtained from the Target database (https://ocg.cancer.gov/programs/target). The public access to the databases is open.

Ethics approval and consent to participate Not applicable.

Consent for publication

Not applicable.

Competing interests

The authors declare that they have no competing interests. 


\section{Author details}

'Department of Ultrasound, Xiaoshan Traditional Chinese Medical Hospital, Hangzhou 311200, China. 'Department of Orthopaedic, Hanchuan People's Hospital, Hanchuan 311200, Hubei Province, China.

\section{Received: 4 June 2020 Accepted: 16 October 2020}

Published online: 21 October 2020

\section{References}

1. Ritter J, Bielack SS. Osteosarcoma. Ann Oncol. 2010;21(Suppl 7):vii320-5.

2. Cui J, Dean D, Hornicek FJ, Chen Z, Duan Z. The role of extracelluar matrix in osteosarcoma progression and metastasis. J Exp Clin Cancer Res. 2020;39:178

3. Duan DD, Xie H, Shi HF, et al. Hint1 overexpression inhibits the cell cycle and induces cell apoptosis in human osteosarcoma cells. Onco Targets Ther. 2020;13:8223-32.

4. Luetke A, Meyers PA, Lewis I, Juergens H. Osteosarcoma treatment - where do we stand? A state of the art review. Cancer Treat Rev. 2014;40:523-32.

5. Zhang $\mathrm{C}$, Zheng $\mathrm{JH}$, Lin ZH, et al. Profiles of immune cell infiltration and immune-related genes in the tumor microenvironment of osteosarcoma. Aging. 2020;12:3486-501.

6. Huang H, Han Y, Chen Z, et al. ML264 inhibits osteosarcoma growth and metastasis via inhibition of JAK2/STAT3 and WNT/beta-catenin signalling pathways. J Cell Mol Med. 2020;24:5652-64.

7. Yang JS, Lin CW, Hsieh YS, et al. Selaginella tamariscina (Beauv.) possesses antimetastatic effects on human osteosarcoma cells by decreasing MMP-2 and MMP-9 secretions via p38 and Akt signaling pathways. Food Chem Toxicol. 2013;59:801-7.

8. Saraf AJ, Fenger JM, Roberts RD. Osteosarcoma: accelerating Progress makes for a hopeful future. Front Oncol. 2018;8:4

9. Lettieri CK, Appel N, Labban N, Lussier DM, Blattman JN, Hingorani P. Progress and opportunities for immune therapeutics in osteosarcoma. Immunotherapy. 2016;8:1233-44.

10. Langley RR, Fidler IJ. The seed and soil hypothesis revisited--the role of tumor-stroma interactions in metastasis to different organs. Int J Cancer 2011;128:2527-35.

11. Petitprez F, Vano YA, Becht E, et al. Transcriptomic analysis of the tumor microenvironment to guide prognosis and immunotherapies. Cancer Immunol limmunother. 2018;67:981-8.

12. Zhou ZH, Ji CD, Xiao HL, Zhao HB, Cui YH, Bian XW. Reorganized collagen in the tumor microenvironment of gastric Cancer and its association with prognosis. J Cancer. 2017:8:1466-76.

13. Angell $H$, Galon J. From the immune contexture to the Immunoscore: the role of prognostic and predictive immune markers in cancer. Curr Opin Immunol. 2013:25:261-7.

14. Zhou R, Zhang J, Zeng D, et al. Immune cell infiltration as a biomarker for the diagnosis and prognosis of stage I-III colon cancer. Cancer Immunol Immunother. 2019;68:433-42.

15. Wolf-Dennen K, Gordon N, Kleinerman ES. Exosomal communication by metastatic osteosarcoma cells modulates alveolar macrophages to an M2 tumor-promoting phenotype and inhibits tumoricidal functions. Oncoimmunology. 2020;9:1747677.

16. Yahiro $\mathrm{K}$, Matsumoto $\mathrm{Y}$, Yamada $\mathrm{H}$, et al. Activation of TLR4 signaling inhibits progression of osteosarcoma by stimulating CD8-positive cytotoxic Iymphocytes. Cancer Immunol Immunother. 2020;69:745-58.

17. Liu P, Xiao Q, Zhou B, Dai Z, Kang Y. Prognostic significance of programmed death ligand 1 expression and tumor-infiltrating lymphocytes in axial osteosarcoma. World Neurosurg. 2019;129:e240-54.

18. Edgar R, Domrachev M, Lash AE. Gene expression omnibus: NCBI gene expression and hybridization array data repository. Nucleic Acids Res. 2002;30:207-10.

19. Paoloni M, Davis S, Lana S, et al. Canine tumor cross-species genomics uncovers targets linked to osteosarcoma progression. BMC Genomics. 2009;10:625.

20. Buddingh EP, Kuijjer ML, Duim RA, et al. Tumor-infiltrating macrophages are associated with metastasis suppression in high-grade osteosarcoma: a rationale for treatment with macrophage activating agents. Clin Cancer Res. 2011;17:2110-9.

21. Kelly AD, Haibe-Kains B, Janeway KA, et al. MicroRNA paraffin-based studies in osteosarcoma reveal reproducible independent prognostic profiles at 14q32. Genome Med. 2013:5:2.

22. Liu Z, Zhu Y, Xu L, et al. Tumor stroma-infiltrating mast cells predict prognosis and adjuvant chemotherapeutic benefits in patients with muscle invasive bladder cancer. Oncoimmunology. 2018;7:e1474317.
23. Mony JT, Schuchert MJ. Prognostic implications of heterogeneity in intratumoral immune composition for recurrence in early stage lung Cancer. Front Immunol. 2018;9:2298.

24. Xiong $Y$, Wang $K$, Zhou H, Peng L, You W, Fu Z. Profiles of immune infiltration in colorectal cancer and their clinical significant: a gene expression-based study. Cancer Med. 2018;7:4496-508.

25. Mao $Y$, Feng $Q$, Zheng $P$, et al. Low tumor infiltrating mast cell density confers prognostic benefit and reflects immunoactivation in colorectal cancer. Int J Cancer. 2018;143:2271-80.

26. Desmedt $C$, Salgado $R$, Fornili $M$, et al. Immune infiltration in invasive lobular breast Cancer. J Natl Cancer Inst. 2018;110:768-76.

27. Abreu-Sánchez A, Ruiz-Castillo J, Onieva-Zafra MD, Parra-Fernández ML, Fernández-Martínez E. Interference and Impact of Dysmenorrhea onthe Life of Spanish Nursing Students. Int J Environ Res Public health. 2020;17:E6473.

28. Zamaninour N, Pazouki A, Kermansaravi M, Seifollahi A, Kabir A. Changes in body composition and biochemical parameters following laparoscopic one anastomosis gastric bypass: 1-year follow-up. Obes Surg. 2020.

29. Benjamens S, Rijkse E, Te Velde-Keyzer CA, et al. Aorto-iliac artery calcification prior to kidney transplantation. J Clin Med. 2020;9.

30. Park C, Kim JH, Kim PH, et al. Imaging predictors of survival in patients with single small hepatocellular carcinoma treated with Transarterial chemoembolization. Korean J Radiol. 2020.

31. Li J, Wang W, Xia P, et al. Identification of a five-IncRNA signature for predicting the risk of tumor recurrence in patients with breast cancer. Int J Cancer. 2018;143:2150-60.

32. Hajalsiddig TTH, Osman ABM, Saeed AEM. 2D-QSAR modeling and molecular docking studies on 1H-Pyrazole-1-carbothioamide derivatives as EGFR kinase inhibitors. ACS Omega. 2020;5:18662-74.

33. Ju M, Qi A, Bi J, et al. A five-mRNA signature associated with posttranslational modifications can better predict recurrence and survival in cervical cancer. J Cell Mol Med. 2020.

34. Chen $Y, B i F, A n Y$, Yang Q. Identification of pathological grade and prognosis-associated IncRNA for ovarian cancer. J Cell Biochem. 2019;120: 14444-54.

35. Fridman WH, Pages F, Sautes-Fridman C, Galon J. The immune contexture in human tumours: impact on clinical outcome. Nat Rev Cancer. 2012;12: 298-306.

36. Hong W, Yuan H, Gu Y, et al. Immune-related prognosis biomarkers associated with osteosarcoma microenvironment. Cancer Cell Int. 2020;20:83.

37. Deng $C, X u Y, F u J$, et al. Reprograming the tumor immunologic microenvironment by neoadjuvant chemotherapy in osteosarcoma. Cancer Sci. 2020.

38. Morrison C. Immuno-oncologists eye up macrophage targets. Nat Rev Drug Discov. 2016:15:373-4.

39. Zhang Y, Morgan R, Podack ER, Rosenblatt J. B cell regulation of anti-tumor immune response. Immunol Res. 2013;57:115-24.

40. Affara NI, Ruffell B, Medler TR, et al. B cells regulate macrophage phenotype and response to chemotherapy in squamous carcinomas. Cancer Cell. 2014; 25:809-21.

41. Lee HW, Choi HJ, Ha SJ, Lee KT, Kwon YG. Recruitment of monocytes/ macrophages in different tumor microenvironments. Biochim Biophys Acta. 2013;1835:170-9.

42. Bell $D$, Berchuck $A$, Birrer $M$, et al. Integrated genomic analyses of ovarian carcinoma. Nature. 2011:474:609-15.

43. Tuohy JL, Lascelles BD, Griffith EH, Fogle JE. Association of Canine Osteosarcoma and Monocyte Phenotype and chemotactic function. J Vet Intern Med. 2016:30:1167-78.

44. Cersosimo F, Lonardi S, Bernardini G, et al. Tumor-associated macrophages in osteosarcoma: from mechanisms to therapy. Int J Mol Sci. 2020;21.

45. Hu X, Li YQ, Li QG, Ma YL, Peng JJ, Cai SJ. Baseline peripheral blood leukocytosis is negatively correlated with T-cell infiltration predicting worse outcome in colorectal cancers. Front Immunol. 2018;9:2354.

46. Tian W, Wang L, Yuan $L$, et al. A prognostic risk model for patients with triple negative breast cancer based on stromal natural killer cells, tumorassociated macrophages and growth-arrest specific protein 6. Cancer Sci. 2016:107:882-9.

\section{Publisher's Note}

Springer Nature remains neutral with regard to jurisdictional claims in published maps and institutional affiliations. 